Bangladesh J Med Microbiol 2021; 15 (2): 1-4

Bangladesh Society of Medical Microbiologists

\title{
Editorial
}

\section{Fleming's Warning and the future pandemic}

\section{Sanya Tahmina Jhora ${ }^{1}$}

${ }^{1}$ Directorate General of Health Services, Dhaka, Bangladesh.

\section{Introduction}

"Antibiotic resistance occurs when bacteria change in response to the use of these medicines. Bacteria, not humans or animals, become antibiotic-resistant"1.

\section{History}

The discovery of Penicillin heralded the dawn of the antibiotic age which was incidentally discovered by Alexander Fleming ${ }^{2}$. The period was 1928. The first antibiotic came for therapeutic purpose by D-Day ${ }^{3}$ in 1944 which saved many lives for infections of injured soldiers of World War II, both in the field and in hospitals throughout Europe. By the end of WWII, for public use, it came in market in $1945^{2}$. Howard Walter Florey and Ernst Boris Chain, sharing with Alexander Fleming won the 1945 Nobel Prize in Physiology or Medicine. Fleming warned of bacterial resistance in his acceptance speech. He said, "The time may come when penicillin can be bought by anyone in the shops. Then there is the danger that the ignorant man may easily underdose himself and by exposing his microbes to non-lethal quantities of the drug make them resistant".

People does not care of this warning. Penicillin and the other antibiotics which were discovered later, have been used indiscriminately, leading to increasing selective pressure and increasing antimicrobial resistance against common pathogens.

While antimicrobial resistance (AMR) is a natural process in bacteria, antibiotic use makes it worse. Bacteria can become resistant to antibiotics when they: (i) turn on certain internal resistance processes, (ii) change to protect themselves from an antibiotic, (iii) receive resistant genes from other bacteria by vertical and horizontal transfer ${ }^{5}$.
The more we use antibiotics, the more chances bacteria will become resistant to them. If we decrease antibiotic use, the antibiotics may again become effective at killing bacteria $^{6}$. It is noteworthy that antibiotic use is not the only factor in applying selective pressure. The use of disinfectants, biocides and the presence of environmental pollutants can also keep the prevalence of resistance high, even if the abuse of antibiotics is restrained ${ }^{7}$.

\section{Particular Challenges for Developing Countries}

The challenges of AMR have different manifestations in developing countries. In developing countries, multi-resistant salmonellosis, shigellosis, and tuberculosis (TB) in the community setting and enteric bacteria and Pseudomonas aeruginosa infections in hospitals are much bigger problem than MRSA problems in developed countries. This statement may give the false impression that resistance affects developed and developing countries equally, but only through different bugs. In reality, the pooled overall health care-associated infection density in adult intensive-care units was at least three times as high in these countries compared to densities reported from the USA ${ }^{8}$. Moreover, most developing countries do not have pharmaceutical research of their own and are therefore dependent on what developed countries study and what drugs they develop. While new anti-staphylococcal drugs are being deployed (e.g. daptomycin, newer glycopeptides), drugs that can be used against multidrug-resistant and extensively drug-resistant (XDR) TB, multidrug-resistant Shigella and Salmonella strains, or multidrug-resistant enteric bacteria causing nosocomial infections are not being fully explored. Very few new antibiotics will be developed in the short and mid-term; recent genomic evidence even suggests we may already have as many antibiotics as is possible ${ }^{9}$. 


\section{Bangladesh situation}

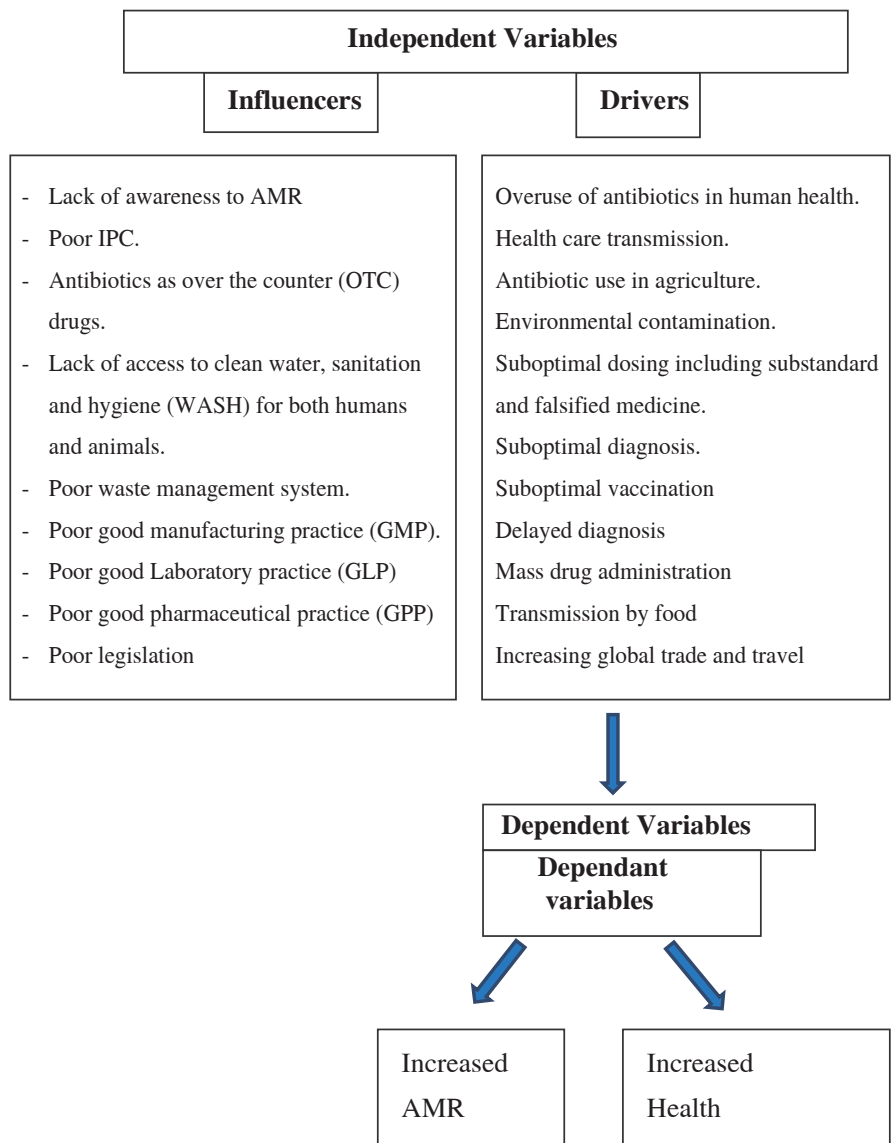

In one study from 2015 to 2019 presenting to clinical care in Bangladesh, 430 bacterial isolates from patients with respiratory, intestinal, wound infections and typhoid fever, were examined. $53 \%$ of the isolates were multidrugresistant (MDR), including 97\% of Escherichia coli. There was a year-wise gradual increase in MDR isolates from 2015 to 2018, and there was an almost twofold increase in the number of MDR strains isolated in 2019 (P $=0.00058)^{10}$.

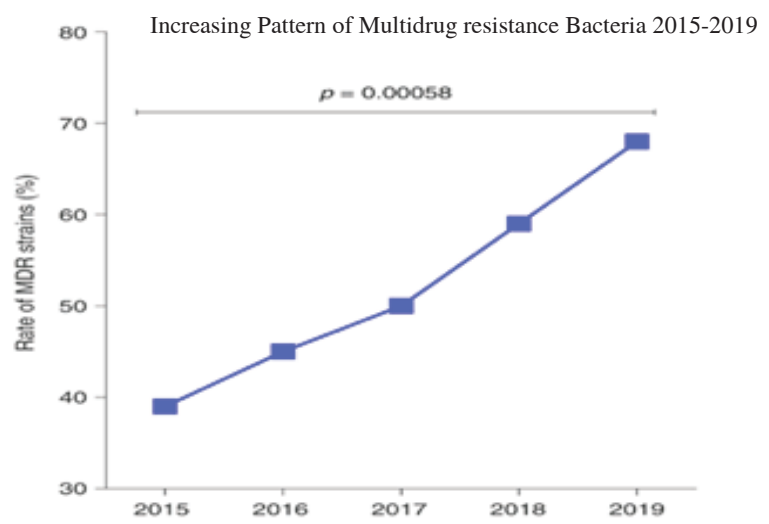

One study in a tertiary hospital from January 2020 to March 2021 shows that $50 \%$ of the isolated organisms are resistant to eight commonly used antibiotics. The six antibiotics which showed $70 \%$ to $90 \%$ sensitivity were all injectable and costly.

Situation in Intensive Care Unit (ICU) is threatening. Institute of Epidemiology, Disease Control and Research (IEDCR) conducted a AMR surveillance across the country during March 2017 to March 2020. Endotracheal aspirate was taken from the ICU patients where more than $60 \%$ of the three identified pathogens (E. coli, K. pneumoniae and $P$. aeruginosa) showed high resistance to all the antibiotics. Acinetobacter complex showed more than $80 \%$ resistance to all the used antibiotics. All the three pathogens, showed more than $90 \%$ resistance to cefepime, a reserve group antibiotic, indicating very alarming but frequently observed scenario in ICU settings ${ }^{11}$. Poor infection prevention and control, indiscriminate use of relatively new antibiotics makes the ICU a warehouse of multidrug resistant strains of common antibiotics leading to development of Superbugs.

The problem is silent, but from above studies, the seriousness could be understood. Due to competitive priority of the acute infectious diseases, different epidemics and pandemic, natural disasters, migrations etc., the magnitude of the problem is often blurred.

COVID Pandemic and Antibiotic misuse: The indiscriminate use of different antimicrobials during the COVID-19 pandemic is huge in Bangladesh. Perhaps it has superseded the previous records of antimicrobial consumption. There is no available data of the antimicrobial consumption during 2020 and 2021. A previous study 'Establishment of a National Antimicrobial Consumption (AMC) Monitoring System in Bangladesh,' showed antibiotic consumption was $16.65 \%$ in Bangladesh in 2016 which has increased to $19.8 \%$ in 2017 and $21.8 \%$ in 2018. The consumption further increased to $25 \%$ till June 2019. During COVID-19 pandemic, Ivermectin, Doxycycline, Azithromycin are being used for any respiratory symptoms, even sometimes these are used for prevention of COVID-19. The antivirals are also used in case of hospitalized patients with or without indications ${ }^{12}$.

Policies of Bangladesh to combat AMR: National Drug Policy 2016 has been introduced but 'National Antibiotic or Antimicrobial Policy' is yet to be introduced. The 
number of registered pharmacies in Bangladesh is around one lakh twenty-five thousand. Beside these there are thousands of Medicine shops which do not maintain any regulations. Even the registered pharmacies sell WATCH group antibiotics without prescriptions. One study showed, the 'WATCH' group of antibiotics are more used than 'ACCESS' group, which is alarming in Bangladesh. It also indicates the inadequate monitoring of the import by the regulatory authority. The study also compared the scenario with African countries where similar type of study showed that 'ACCESS' group are used more than the 'WATCH' group"13.

Recently 668 Pharmacies and 41009 medicine shops have been upgraded by DGDA as 'Model Pharmacies' and 'Model Medicine Shops' which are insignificant in comparison to the large market. Specific laws on different aspects of food animals and fish were developed in 2010 where the use of antimicrobials, growth hormones, and pesticides were banned. But due to weak monitoring, it is difficult to assess how these are being maintained.

Bangladesh, being a signatory of the "Jaipur Declaration2011" for the containment of antimicrobial resistance, has responded to AMR by taking an initiative to implement integrated AMR activities through a multi-sectoral approach. The Communicable Disease Control (CDC) program of Directorate General of Health Services (DGHS) of Bangladesh is the national focal point of ARC. Antimicrobial resistance Containment (ARC) program has been incorporated in the $4^{\text {th }}$ Health Population and Nutrition Sector Program (HPNSP) in 2017.

National strategy (NSP) for antimicrobial resistance Containment (ARC) in Bangladesh 2011-2016 and National Action Plan (NAP) 2017-2022 have been approved by the Ministry of Health and Family Welfare. Recently the NSP and NAP of 2021-2026 have been developed under the guidance of Global Action Plan (GAP). The main focus of the national strategy for ARC are to: strengthen multi-sectoral coordination in one health approach; increase awareness and understanding of AMR through advocacy communication and educations; establish integrated surveillance and strengthen laboratory capacity; reduce the incidence of infection through effective sanitation, hygiene and infection prevention control (IPC) measures and good agricultural and biosecurity practices; promote antimicrobial stewardship programs in human and animal health sectors; reduce environmental spread of
AMR; promote innovation and research on AMR; strengthen global collaboration and partnerships.

\section{Conclusion}

The problem is silent and if proper steps will not be taken immediately, it may produce more dangerous situation than COVID-19. The Global Leaders Group (GLG) on Antimicrobial Resistance (AMR) has been formed in 2020 to strengthen global political momentum and leadership on AMR, co-chaired by Honorable PM Sheikh Hasina and Mia Amor Mottley, Prime Minister of Barbados. This type of initiative in the midst of the pandemic indicates how the global leaders are concerned about the danger of AMR.

During the launching ceremony of One Health Global Group on AMR, the DG of WHO warned, "Antimicrobial resistance may not seem as urgent as a pandemic, but it is just as dangerous. It threatens to slow down a century of medical progress and leave us defenseless against infections that today can be treated easily".

\section{References}

1. World antibiotic awareness week, November 18-24, 2022. Natonal Today. 2021 Nov 18.

2. Gaynes R. The discovery of penicillin-new insights after more than 75 years of clinical use. Emerging infectious diseases. 2017 May;23(5):849.

3. D-Day-Invasion, Facts \& Significance. History [Updated 2019 Jun 5; Cited 2021 Nov 8]. Available at: https://www.history.com > topics > world-war-ii >d-day.

4. Alexander Fleming- Penicillin. Nobel Lecture. [Cited 2021 Nov 8]. Available at: https://www.nobel prize.org > uploads > 2018/06.

5. Antimicrobial resistance. Australian Government [Cited 2021 Nov 5]. Available at: https://ww w.amr.gov.au/about-amr/what-causes-amr.

6. What causes AMR? - Antimicrobial resistance. Australian Government [Cited 2021 Nov 5]. Avail ableat:https://www.amr.gov.au/about-amr/ what-causes-amr.

7. Korves T, Garay C, Carleton HA, Sabol A, Trees E, Peterson MW. Assessing Pathogens for Natural versus Laboratory Origins Using Genomic Data and Machine Learning. Biorxiv. 2016 Jan 1:079541. 
8. Allegranzi B, Nejad SB, Combescure C, Graafmans W, Attar H, Donaldson L, et al. Burden of endemic health-care-associated infection in developing countries: systematic review and meta-analysis. The Lancet. 2011 Jan 15;377(9761):228-41.

9. Sosa AJ, Byarugaba DK, Anabile-Cuevas CF, Hsueh P, Kariuki S, Okeke IN. Antimicrobial Resistance in Developing Countries. New York: Springer; 2010.p.10.

10. Safain KS, Bhuyan GS, Hasib SH, Islam MS, Al Mahmud-Un-Nabi M, Sultana R, et al. Genotypic and phenotypic profiles of antibiotic-resistant bacteria isolated from hospitalised patients in Bangladesh. Tropical medicine \& international health: TM \& IH. 2021 May 2.
11. Habib ZH, Alam MA, Bably NN, Khan IA, Rizvi SS, Shirin T, et al. The findings of Antimicrobial Resistance Surveillance in Bangladesh (2016-2020). medRxiv. 2021 Jan 1.

12. Antibiotic consumption increased by $30.81 \%$ in two years: Study. TBS Report. 2021 Nov 28.

13. WHO releases the 2019 Aware Classification Antibiotic. World Health Organization [Updated 2019 Oct; Cited 2021 Nov 12]. Available at: https://www.who. int $>$ medicines $>$ news. 